\title{
Spatial and temporal variations of antibiotics in a tidal river
}

\author{
Jia Li • Min Cui • Hua Zhang
}

Received: 2 December 2019 / Accepted: 21 April 2020

(C) Springer Nature Switzerland AG 2020

\begin{abstract}
Estuary is an important route for the transport of terrestrial contaminants to the ocean. Its unique hydrodynamic properties may influence the fate and distribution of pollutants. Previous studies have shown that severe pollution because of antibiotics has occurred in many inland surface waterbodies; however, the behavior of antibiotic residuals remains poorly understood in estuarine environments. In this study, the occurrence and spatiotemporal distribution of seven selected antibiotics (i.e., sulfamethazine, sulfamethoxazole, trimethoprim, ofloxacin, ciprofloxacin, erythromycin, and roxithromycin) in a tidal river were investigated through one continuous and four synoptic sampling events. Results show that the concentrations of most antibiotics are in the nanogram per liter level, except for trimethoprim with the highest concentration up to $12,440 \mathrm{ng} \mathrm{L}^{-1}$ during the wet season. Except for sulfamethazine, the other
\end{abstract}

Electronic supplementary material The online version of this article (https://doi.org/10.1007/s10661-020-08313-2) contains supplementary material, which is available to authorized users.

J. Li • M. Cui

School of Environmental Science and Engineering, Yangzhou University, Yangzhou 225127, China

J. Li $(\bowtie) \cdot$ H. Zhang $(\bowtie)$

Key Laboratory of Coastal Environmental Process and Ecology Remediation, Shandong Key Laboratory of Coastal Environmental Processes, Yantai Institute of Coastal Zone Research, Chinese Academy of Sciences (YICCAS),

Yantai 264003, China

e-mail: lijia3611@yzu.edu.cn

e-mail: hzhang@yic.ac.cn six antibiotics showed high concentrations (i.e., > $100 \mathrm{ng} \mathrm{L}^{-1}$ ) in at least one sampling campaign. Different temporal distribution patterns of these antibiotics indicated that they were mainly controlled by source loading, flow condition, and discharge amounts. Spatial distribution indicated that the main pollution source of trimethoprim was located in lower reaches, while the other six antibiotics mainly came from the upstream sources. Based on the theoretical dilution line, erythromycin and roxithromycin degraded in the tidal river, whereas the other five types of antibiotics showed a conservative behavior. Tide has important effects on the spatial distribution of antibiotics, especially those with a wide concentration range, in estuarine environments. Furthermore, risk assessment based on the calculated risk quotients showed that five types of antibiotics pose high risks to aquatic organisms. These observations provided new insight into the distribution and transport of common antibiotics in estuarine environments.

Keywords Antibiotics · Estuary $\cdot$ Spatiotemporal distributions · Transport · Ecological risk · Tide

\section{Introduction}

Antibiotics play an important role in today's society. Enormous amounts of antibiotics are used for specific purposes such as in the prevention and treatment of diseases and in the preservation of food as well as feed 
additives to promote animal growth (Cromwell 2002; $\mathrm{Hu}$ et al. 2003). In 2013, China used approximately 162,000 tons of antibiotics (Zhang et al. 2015). However, most antibiotics cannot be metabolized completely after entering an organism, and $80-90 \%$ of these antibiotics are excreted via urine and feces before being discharged into aquatic environments (Bound and Voulvoulis 2004). As reported by Zhang et al. (2015), an estimated 24,750 tons of the excreted antibiotics were discharged into the surface water in China per year. Although the concentrations of antibiotics in the surface water are at trace levels, they still pose great threats to human health and the ecosystem (Birch et al. 2015). A direct consequence of antibiotic residues is the occurrence of antibiotic resistance, and antibiotic resistance genes (ARGs) have been detected in aquatic environments (Yang et al. 2017; Zhao et al. 2017; Zheng et al. 2017). More serious is antibiotics and ARGs can get transferred through food webs and may enter human bodies eventually (Liu et al. 2017).

Antibiotics in aquatic environments have multiple sources including the effluents of waste water treatment plants (WWTPs), wastewater discharges from aquaculture and livestock farming, runoff from farmland applied with manure, and leakage discharges from pharmaceutical factory (He et al. 2016; Kim et al. 2017; Cheng et al. 2018). Once entering the surface waters, antibiotic residues may undergo long-range transport. As reported by Biel-Maeso et al. (2018), antibiotics in the effluents of WWTPs can transfer from a river to an estuary and then to bay, and eventually to the open sea. The growing concern in antibiotics over the last decades has resulted in numerous reports of these pollutants in waste water, river, lake, reservoir, and seawater ( $\mathrm{Li}$ et al. 2012; Wolf et al. 2013; Jiang et al. 2014; Kimosop et al. 2016; Li et al. 2016a, b; Vo et al. 2016; Zhang et al. 2018a, b). However, antibiotic residuals and their transport behaviors remain poorly understood in estuarine environments.

Estuary is an important route for the transport of terrestrial material to the ocean (Simpson et al. 2001). Under the combined influences of river runoff and tides, the hydrological properties of an estuary are dynamic and complex. For example, salinity, $\mathrm{pH}$, flow rate, water level, redox potential, and the concentration of particles exhibited high spatial and temporal variability (Chapman and Wang 2001). Furthermore, the flow in estuary was characterized by reciprocating flow; the flow direction also changed irregularly (Zou et al. 2016). The unique hydrological properties may influence the fate and transport of pollutants in estuary. Zhang et al. (2017) demonstrated that antibiotic concentrations in the East River estuary were affected by the tidal change. Furthermore, Zhao et al. (2015) revealed that concentrations of pharmaceuticals in the Yangtze estuary decreased during tidal rise and then increased during tidal receding. Increasing anthropogenic activities around coastal zones resulted in the discharge of excessive pollutants into estuarine environments, posing a great press to their environmental and ecological functions (Zou et al. 2016). Antibiotic residuals from the upper catchment are stranded in the estuary, which make the estuary a pollution hotspot (Biel-Maeso et al. 2018). Thus, it is important to investigate the current status of antibiotic pollution and reveal the transport mechanisms of these antibiotics in estuarine environments.

The Xiaoqing River (XQR) is a major pollution source and the cause of ecological deterioration in Laizhou Bay (LZB) (Luo et al. 2013). The XQR basin is densely populated (Shi et al. 2015). More than 300 million tons of domestic sewage discharge into the $\mathrm{XQR}$ every year. Furthermore, animal husbandry and aquaculture farming in the $\mathrm{XQR}$ basin are intensive ( $\mathrm{Li}$ et al. 2016a, b). These potential pollution sources make XQR a hotspot of antibiotics pollution. Our previous study found that thirteen antibiotics can be detected in the XQR with the highest concentration up to $3900 \mathrm{ng} \mathrm{L}^{-1}$ (Li et al. 2016a, b). These antibiotics could be transported to the semi-enclosed LZB, and inevitably have a negative impact on marine ecological environment (Liu et al. 2017). However, owing to special hydrogeomorphic features such as trumpet mouth, broad intertidal shoal, and gentle slope, pollutants move to sea slowly and are prone to be stranded in the XQR estuary for a long time (Shen et al. 2017).

The objectives of this study were not only to fill up the data gap of the occurrence, spatiotemporal distributions, and ecological risks of antibiotics in the $\mathrm{XQR}$ estuary by analyzing the selected antibiotics in the estuarine water but also to reveal the influences of tide on the distribution and transport of antibiotics in the estuarine environment.

\section{Materials and methods}

Chemicals and standards

The selected antibiotic standards including sulfonamides (sulfamethazine (SMZ) and 
sulfamethoxazole (SMX)), sulfonamide synergists (trimethoprim (TMP)), fluoroquinolones (ofloxacin (OFL) and ciprofloxacin (CIP)), and macrolides (erythromycin (ETM) and roxithromycin (RTM)) were purchased from Sigma-Aldrich Company (USA). The physicochemical properties of the seven antibiotics are shown in Table S1. The target antibiotics were dissolved in methanol (ACS, USA) with a concentration of $50 \mathrm{mg} \mathrm{L}^{-1}$. It should be noted that erythromycin was detected as its dehydration product $\left(\mathrm{ETM}-\mathrm{H}_{2} \mathrm{O}\right)$. The solution of ETM- $\mathrm{H}_{2} \mathrm{O}$ was prepared by acidification using the method by Mcardell et al. (2003). These stock solutions were stored at $4{ }^{\circ} \mathrm{C}$ until used. The standards, sulfadimoxine-d6 (SDM-d6) and simatone (SMT) were obtained from SigmaAldrich Company (USA). Methanol and acetonitrile were HPLC grade and were obtained from Anaqua Chemicals Supply (ACS, USA). Ultrapure water (MQ) was obtained from a Milli-Q water purification system (Millipore, Billerica, MA, USA). The other chemicals and solvents used in this study were of analytical grade or above.
Study area and sample collection

The tidal section of XQR (Fig. 1) starts from Wangdao sluice ( $\left.37^{\circ} 11^{\prime} 14.46^{\prime \prime} \mathrm{N}, 118^{\circ} 35^{\prime} 55.19^{\prime \prime} \mathrm{E}\right)$ and ends at the LZB, with a total length of approximately $50 \mathrm{~km}$. The XQR estuary is a shallow estuarine system (water depth $<8 \mathrm{~m}$ ) and has irregular semidiurnal tides with a tidal form number of $0.5-0.7$ and a tidal range of about $2 \mathrm{~m}$ (Zou et al. 2016). The average annual rainfall of this area is $646.7 \mathrm{~mm}$, and the rainfall is mostly concentrated from June to September. Solar radiation is mainly concentrated in the summer (June-September), accounting for about $30-33 \%$ of the total annual radiation.

A total of 10 sampling sites (A1-A10) with an average interval of $5 \mathrm{~km}$ were set along the XQR estuary (Fig. 1). Detailed information about the sampling sites is listed in Table S2. During 2015 to 2017, five sampling campaigns were conducted, which included four synoptic samplings along the XQR estuary (i.e., sites A1A10) and a continuous sampling at a fixed site (i.e., site A6) lasted for two tidal cycles (25 h) during May 16-17, 2017. Surface water samples were collected at 0-20 cm depth using a stainless steel bucket. Sodium azide was

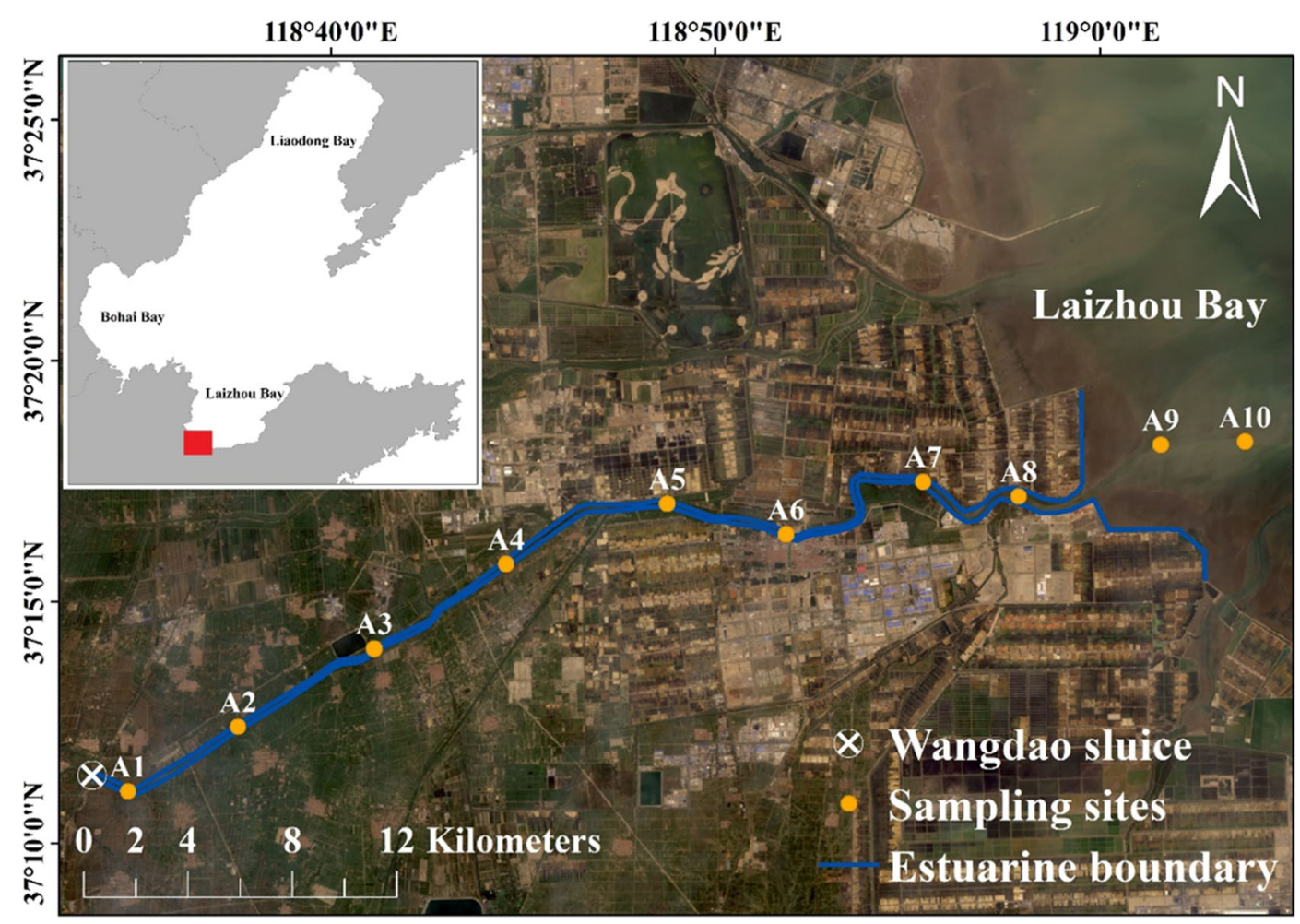

Fig. 1 Map of sampling sites in the Xiaoqing River estuary 
added in situ to inhibit the microbial activity. Thereafter, water samples were collected into clean amber glass bottles and stored at $4{ }^{\circ} \mathrm{C}$ during transport to the laboratory, where the samples were processed within $48 \mathrm{~h}$.

\section{Sample extraction}

The antibiotics in the water were concentrated using the solid-phase extraction (SPE) method. All water samples were filtered through $0.45-\mu \mathrm{m}$ cellulose acetate membranes. Then, $0.5 \mathrm{~L}$ water sample was acidified to $\mathrm{pH}=$ 3.0 using sulfuric acid solution $\left(3 \mathrm{~mol} \mathrm{~L}^{-1}\right)$, followed by the addition of $0.2 \mathrm{~g}$ disodium ethylenediaminetetraacetic acid (Na2EDTA) and $100 \mathrm{mg}$ SDM-d6, which was used as a surrogate standard. The pretreated water sample was concentrated by passing through the Oasis HLB cartridge (200 mg, waters) at an approximate flow rate of $5.0 \mathrm{~mL} \mathrm{~min}{ }^{-1}$. The HLB cartridge was preconditioned with $6 \mathrm{~mL}$ methanol and $6 \mathrm{~mL}$ ultrapure water $(\mathrm{pH}=3.0)$ with a flow rate of approximately $2.0 \mathrm{ml} \mathrm{min}{ }^{-1}$. After the water had passed through the combined cartridges, the HLB cartridge was rinsed with $10 \mathrm{~mL}$ of ultrapure water and dried for $30 \mathrm{~min}$. Then each cartridge was eluted sequentially with $2.0 \mathrm{~mL}$ methanol for three times at a rate of $<1.0 \mathrm{~mL} \mathrm{~min}{ }^{-1}$. The analytes were collected in a $10-\mathrm{mL}$ brown glass vial, and the eluent was condensed to near dryness under a gentle stream of nitrogen at $35^{\circ} \mathrm{C}$. Before analysis, $100 \mathrm{ng}$ of internal standard (SMT) was added, and the final volume in each vial was adjusted to $1.0 \mathrm{~mL}$ accurately with methanol/ultrapure water $(6: 4 \mathrm{~V} / \mathrm{V})$.

Analysis conditions

Antibiotics were analyzed using a high-performance liquid chromatograph (HPLC) system (Thermo Fisher, USA) coupled with TSQ Quantum Access MAX triple quadrupole mass spectrometer (Thermo Fisher, USA). The target antibiotics in samples were separated using a Waters SunFire C18 column $(2.1 \times 150 \mathrm{~mm}, 3.5 \mu \mathrm{m}$ particles) at a constant temperature of $30^{\circ} \mathrm{C}$. Chromatographic analyses were carried out with a mobile phase consisting of water with $0.1 \%$ formic acid (mobile phase A) and methanol (mobile phase B) at a flow rate of $300 \mu \mathrm{L} \mathrm{min}{ }^{-1}$. The elution gradient is listed in Table S3. Mass spectrometric analyses were performed using ESI in positive ionization mode. Data acquisition was carried out in the multiple reaction monitoring
(MRM) mode. The optimal conditions for the analyte monitoring are summarized in Table S4.

Quality analysis and quality control

Quantification of targeted antibiotics was performed using an internal standard calibration curve method established by Luo et al. (2011). The $R^{2}$ values of standard calibration curve for all seven antibiotics were greater than 0.99 (Table S5). The limit of detection (LOD) and the limit of quantification (LOQ) were defined as the concentration corresponding to signal-tonoise $(\mathrm{S} / \mathrm{N})$ ratios of 3 and 10 , respectively. The LOD and LOQ for targeted antibiotics ranged from 0.2 to $0.9 \mathrm{ng} \mathrm{L}^{-1}$ and 0.7 to $2.8 \mathrm{ng} \mathrm{L}^{-1}$, respectively (Table S5). For the recovery experiments, $0.5 \mathrm{~L}$ of filtered estuarine water $(n=3)$ fortified with $50 \mathrm{ng}$ of target antibiotics was treated using the same procedure as the field samples. The average recoveries of the targeted antibiotics ranged from 64.6 to $89.5 \%$ (Table S5). Reported concentration data were not corrected for recovery. All equipment and containers were pre-cleaned using methanol to minimize pollution during experiments. Instrumental blanks (methanol only) were analyzed for every 10 samples to monitor the instrumental background. The procedural blanks prepared with Milli-Q water were extracted following the same procedures as the field samples. The concentrations of all targeted antibiotics in the blank samples were below the LOQ.

Risk assessment

According to the European technical guidance (EC 2003), risk quotients (RQs) were calculated to evaluate the potential ecological risks of antibiotics at different sampling events. The equation is as follows:

$\mathrm{RQ}=\frac{\mathrm{MEC}}{\mathrm{PNEC}}$

where MEC is the measured environmental concentration of each antibiotic; PNEC is predicted no-effect concentration and derived from chronic and acute toxicity data available in the literatures. The calculation of PNEC is obtained from the toxicity data divided by an assessment factor. If only short-term/acute toxicity data EC50/LC50 are available, the assessment factor is 1000 . If the long-term/chronic NOEC values for one, two, or three trophic levels are available, an assessment factor of 
100,50 , or 10 is used (EC 2003). However, an assessment factor of 1000 was also used for the long-term/ chronic toxicity data in some studies (Isidori et al. 2005). The PNEC values were listed in Table S6.

\section{Statistical analysis}

Data analysis was performed using Microsoft Excel 2016 and OriginPro 2015 (OriginLab Corporation, Northampton, MA). Statistical analysis was performed using the SPSS software (Version 19.0, IBM-SPSS, Chicago, IL, USA). Before the analysis, the concentration values were set to half of LODs if antibiotics were not detected. An analysis of variance (ANOVA) was performed for the seven antibiotics between the four sampling campaigns and the significance level was set at $p<0.05$. Non-parametric correlations (Spearman's rank) were calculated between antibiotic concentrations and water level with a significance level set at $p<0.05$.

\section{Results and discussion}

Occurrence of antibiotics in the XQR estuary

As summarized in Table 1, all seven antibiotics had a detection rate of $>80 \%$ in the surface water throughout the four synoptic sampling campaigns along the XQR estuary. It is reasonable because the seven targeted antibiotics were selected based on the investigation results of the XQR basin ( $\mathrm{Li}$ et al. 2016b). The high detection rates of these antibiotics can be linked to their large consumption and/or their refractory behavior in natural aquatic environments. As reported by Zhang et al. (2015), fluoroquinolones and macrolides were two kinds of the most commonly used antibiotics in North China. Sulfonamides were widely detected in aquatic environments of North China (Luo et al. 2011; Zou et al. 2011; Li et al. 2012; Zhang et al. 2012) because of their low affinity to sediment (Spielmeyer et al. 2017) and low degradation rates (Zheng et al. 2012). Furthermore, previous study has demonstrated that TMP was stable in seawater (Lunestad et al. 1995).

In general, the selected antibiotic concentrations ranged from nd (not detected) to $12,440 \mathrm{ng} \mathrm{L}^{-1}$ (Table 1). The coefficients of variation (CV) of RTM were $<1.0$, implying that the concentration of RTM in the study area was not highly variable. On the contrary, the concentrations of the other antibiotics were significantly variable along the XQR estuary, as their $\mathrm{CV}$ values were $>1.0$. Except for SMZ, the other six antibiotics showed high concentrations $\left(>100 \mathrm{ng} \mathrm{L}^{-1}\right)$ in at least one sampling campaign. The mean concentrations of the seven antibiotics follow the order as: TMP $>$ CIP $>$ ETM $>$ SMX $>$ RTM $>$ OFL $>$ SMZ . Among all the antibiotics, TMP exhibited the highest concentrations, which were 2 or 3 orders higher than the maximum concentration of the other antibiotics. Its concentration in the XQR estuary is also much higher than that in other estuaries around the world (Table S7), and even some wastewaters of hospital and effluents of WWTPs (Loos et al. 2013; Vo et al. 2016; Ngigi et al. 2020). Therefore, the high level of TMP in the XQR estuary may be related to the discharge of some pharmaceutical production wastewater. A pharmaceutical factory named Fulkon is located $40 \mathrm{~km}$ from the XQR estuary. This factory is the world's largest producer and exporter of TMP. Its subsidiary is located only $8.5 \mathrm{~km}$ from the study area. However, this is only a reasonable inference, and it needs to be proved based on pollution source investigation in the future.

A global comparison of the selected antibiotic concentrations in estuary is shown in Table S7. The concentration of SMZ in the XQR estuary was lower than those reported in the Pearl River estuary, Yangtze estuary, and Haihe estuary but higher than those in the Yellow River estuary, Jiulong River estuary, Guangli River estuary, and Seine River estuary. The concentration of ETM was comparable with those in the Pearl River estuary and Yangtze estuary, but it was higher than those in the Guangli River estuary, Yellow River estuary, Dalang River estuary, Dafeng River estuary, Danshuei River Estuary, Ebro River estuary, and three estuaries in the UK. Compared with those reported in the other thirty estuaries from seven countries, the concentrations of SMX, TMP, CIP, OFL, and RTM in the XQR estuary were much higher (Table S7). Overall, our results demonstrated that pollution caused by the seven antibiotics in the XQR estuary was more serious than the other estuaries in the world.

Temporal distribution of antibiotics in the XQR estuary

One-way ANOVA indicated that the concentrations of most antibiotics were significantly different between the four sampling campaigns $(p<0.05)$, except for SMX and RTM. From May 27, 2015, to March 29, 2017, the average concentration of 
Table 1 Statistics of antibiotic concentrations in surface water of the XQR estuary during four synoptic sampling campaigns

\begin{tabular}{|c|c|c|c|c|c|c|c|}
\hline Statistics & SMZ & SMX & TMP & CIP & OFL & ETM & RTM \\
\hline \multicolumn{8}{|c|}{ May $27,2015(n=10)$} \\
\hline Range (ng L ${ }^{-1}$ ) & $5.1-12.7$ & $12.3-313$ & $192-4067$ & nd-21.8 & $1.0-44.0$ & $11.9-223$ & $2.3-177$ \\
\hline Average (ng L $\left.{ }^{-1}\right)$ & 9.6 & 112 & 1639 & 15.6 & 11.1 & 143 & 88.3 \\
\hline $\mathrm{SD}$ & 2.6 & 113 & 1445 & 5.9 & 12.9 & 60.2 & 61.2 \\
\hline $\mathrm{CV}$ & 0.3 & 1.0 & 0.9 & 0.4 & 1.2 & 0.4 & 0.7 \\
\hline DR $(\%)$ & 100 & 100 & 100 & 90 & 100 & 100 & 100 \\
\hline \multicolumn{8}{|c|}{ September 13, $2015(n=9)$} \\
\hline Range (ng L ${ }^{-1}$ ) & nd-9.0 & nd-11.4 & nd- $-12,440$ & nd-21.8 & $0.5-20.9$ & $18.6-181$ & $2.8-178$ \\
\hline Average (ng L ${ }^{-1}$ ) & 5.4 & 8.8 & 3456 & 6.8 & 5.8 & 132 & 85.6 \\
\hline SD & 3.9 & 7.1 & 4440 & 9.7 & 5.9 & 54.6 & 54.7 \\
\hline $\mathrm{CV}$ & 0.7 & 0.8 & 1.3 & 1.4 & 1.0 & 0.4 & 0.6 \\
\hline $\mathrm{DR}(\%)$ & 66.7 & 77.8 & 88.9 & 33.3 & 100 & 100 & 100 \\
\hline \multicolumn{8}{|c|}{ December 6, $2015(n=10)$} \\
\hline Range (ng L ${ }^{-1}$ ) & nd- 6.5 & $3.1-45.8$ & $8.5-62.2$ & $2.1-70.0$ & $2.8-30.8$ & $14.5-55$ & $4.3-94.1$ \\
\hline Average (ng L ${ }^{-1}$ ) & 1.8 & 31.1 & 25.3 & 16.8 & 14.8 & 14.7 & 63.4 \\
\hline SD & 2.3 & 8.1 & 19.7 & 17.4 & 8.4 & 10.6 & 8.4 \\
\hline $\mathrm{CV}$ & 1.3 & 0.3 & 0.8 & 1.0 & 0.6 & 0.3 & 0.6 \\
\hline $\mathrm{DR}(\%)$ & 80 & 100 & 100 & 100 & 100 & 100 & 100 \\
\hline \multicolumn{8}{|c|}{ March 29, $2017(n=10)$} \\
\hline Range (ng L ${ }^{-1}$ ) & $5.7-64.2$ & $15.6-820$ & $2.3-28.6$ & $224-877$ & $17.6-240$ & nd-79.0 & $18.3-144$ \\
\hline Average (ng L $\left.{ }^{-1}\right)$ & 16.8 & 154 & 14.4 & 414 & 90.9 & 22.0 & 108 \\
\hline SD & 18.7 & 246 & 10.9 & 268 & 91.3 & 23.7 & 37.4 \\
\hline $\mathrm{CV}$ & 1.1 & 1.6 & 0.8 & 0.6 & 1.0 & 1.1 & 0.3 \\
\hline DR $(\%)$ & 100 & 100 & 100 & 100 & 100 & 90 & 100 \\
\hline \multicolumn{8}{|l|}{ Total $(n=39)$} \\
\hline Range (ng L ${ }^{-1}$ ) & nd- 64.2 & nd -820 & nd $-12,440$ & nd- -877 & $0.5-240$ & nd-223 & $2.3-178$ \\
\hline Average (ng L $\left.{ }^{-1}\right)$ & 8.5 & 78.3 & 1226 & 125 & 38.2 & 89.1 & 74.0 \\
\hline $\mathrm{SD}$ & 11.3 & 149 & 2651 & 230 & 58.1 & 67.1 & 57.5 \\
\hline $\mathrm{CV}$ & 1.3 & 1.9 & 2.2 & 1.8 & 1.5 & 0.8 & 0.8 \\
\hline DR $(\%)$ & 87.2 & 94.9 & 97.4 & 82.1 & 100 & 97.4 & 100 \\
\hline
\end{tabular}

$S D$, standard deviation; $C V$, coefficient of variation; $D R$, detection rate; $n d$, not detected

TMP increased first and then decreased, while the other six antibiotics showed the opposite trend (Fig. 2). Except for TMP, the average concentrations of the other six antibiotics in September were lower than those in May. This may be attributed to the stronger solar radiation in summer (Vieno et al. 2005; Lindholm-Lehto et al. 2016; Zhao et al. 2015), which will accelerate the photodegradation of these six kinds of antibiotics in September. Another possible explanation is that the point source pollution was dominant for these six antibiotics in the XQR estuary, and river water dilution had a great impact on the concentrations of these antibiotics (Tamtam et al. 2008; Jiang et al. 2011; Sun et al. 2016), because river flow of the XQR in September was larger (Zou et al. 2016). In contrast, TMP was stable under sunlight (Lunestad et al. 1995). Strong solar radiation in September had no significant effect on TMP. Thus, the key reason for the higher concentration of TMP in September may be that the increase of pollution loads due to the increase of runoff. Firstly, large amounts of TMP came from the nonpoint source flowed into the XQR estuary with the flood 
Fig. 2 Average concentrations of antibiotics at different sampling date

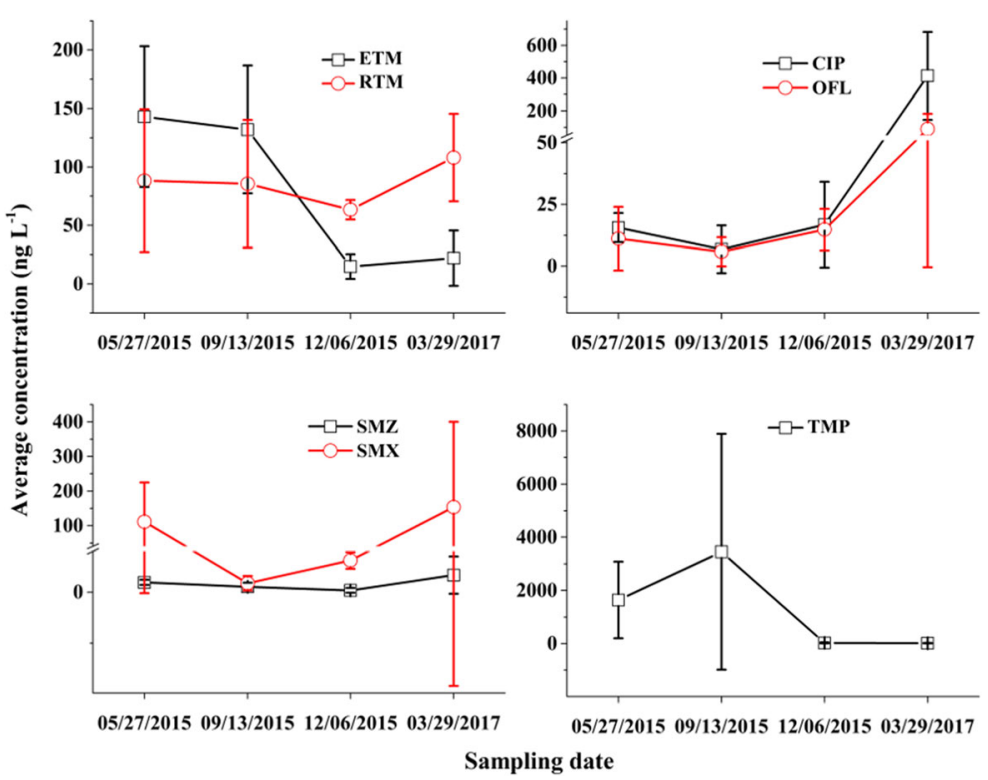

or drainage in September. Secondly, those TMP came from the point source (e.g., tributary) also increased with the increase of runoff from May to September. Correspondingly, reduction of the average concentration of TMP at December 2015 and March 2017 could also be attributed to the decrease in precipitation and/or runoff.

From September to December, the seven antibiotics do not exhibit the same change trend (Fig. 2). The concentrations of CIP, OFL, and SMX increased, while the other four antibiotics decreased. CIP, OFL, and SMX are commonly used to prevent and treat the flu and/or respiratory infections that are more inclined to break out in winter. Therefore, a high consumption of CIP, OFL, and SMX and low water in December may increase their concentrations in December. To maintain the water level of upper reaches of the XQR, the dam of Wangdao is usually turned off in dry season (e.g., December). The reduction of river flow can reduce the pollution loads of antibiotics into the XQR estuary. Correspondingly, the average concentrations of ETM, RTM, and SMZ in December decreased compared with those in May and September (Fig. 2). Meanwhile, the total concentrations of antibiotics in December were the lowest (Fig. 3), which could also be attributed to the reduction of runoff. Once the dam of Wangdao was turned off, antibiotics that came from the upper catchment accumulated here. The retention effect of gate dam on the antibiotics in river has been supported by Guo et al. (2017). When the dam was turned on again, the cumulative river water containing the antibiotics flowed into the XQR estuary, which could have increased the antibiotic pollution. As shown in Fig. 2, the average concentrations of most antibiotics increased in March 2017, except for TMP. This was not surprising because the dam of Wangdao had been just turned on before sampling in March 2017. However, the average concentrations of TMP decreased during flood releasing. This indicated that river discharge was not the main pollution source of TMP in the XQR estuary. Water increased after opening the dam would dilute the TMP and cause a reduction of its concentration. In short, the concentrations of antibiotics varied in different seasons, which can be attributed to the different pollution loads, various hydraulic conditions, and different meteorological conditions.

Spatial distribution of antibiotics in the XQR estuary

Figure 3 illustrates the individual and total concentrations of antibiotics at each sampling site along the tidal river. In December 2015 and March 2017, the total concentrations of the seven antibiotics decreased from the upper estuary to the sea (i.e., A1 to A10). In May and September 2015, the total concentrations of antibiotics in the estuary decreased first, then increased, and then decreased again from A1 to A10. The increase of total antibiotic concentrations in the middle reaches of the estuary (i.e., A4 to A7) was mainly caused by the high content of TMP. If the TMP was excluded, the total 
Fig. 3 Individual concentration of antibiotics along the $\mathrm{XQR}$ estuary from A1 to A10 was in accordance with the direction of that from upper estuary to sea. Data of A8 in September 13, 2015, was missing

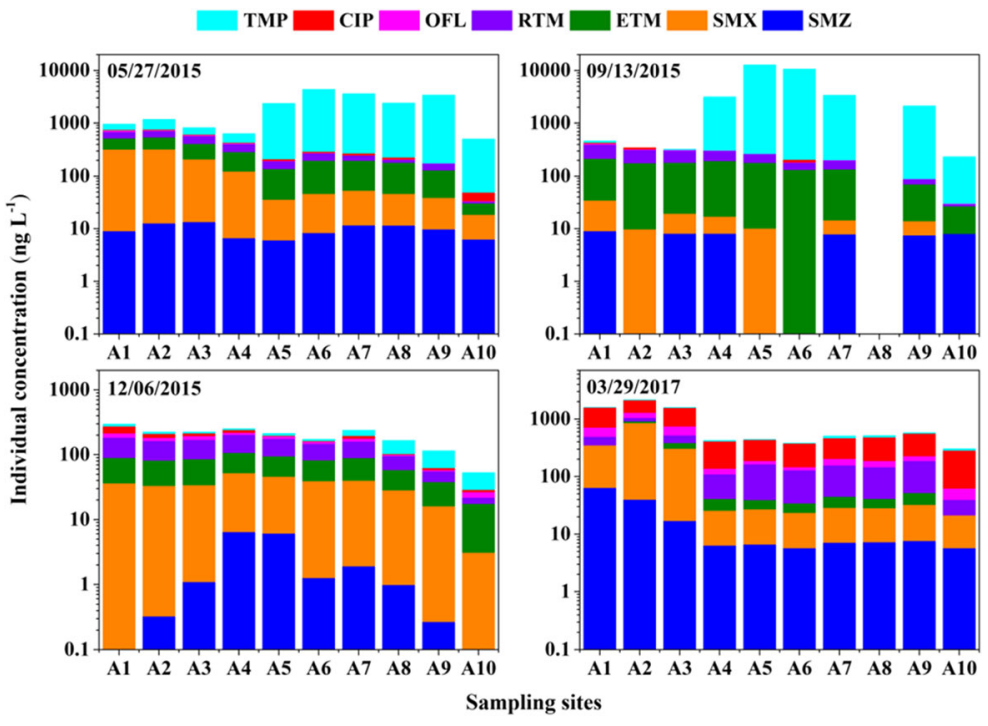

concentrations of the other six antibiotics also decreased from A1 to A10. A similar reduction trend of antibiotics from the upper estuary to the sea was reported in other estuaries (Yan et al. 2013; Sun et al. 2016). The spatial distributions of these six kinds of antibiotics (i.e., SMZ, SMX, ETM, RTM, CIP, and OFL) in the XQR estuary (Fig. 3) implied that they were discharged into the estuary through the river runoff. This also indicated that river runoff was an important source of pollutants in estuaries. These six antibiotic concentrations decreased towards the sea as the river water was diluted by the antibiotic-free seawater. However, one-way ANOVA showed that the variation of spatial distribution patterns of these six antibiotics was not significant $(p>0.05)$. Furthermore, the decreased trend of these six antibiotics was discrete. As illustrated in Fig. 3, a low decrease or an increase of the total antibiotic concentrations was observed at sites A6-A7 in May 2015, and at site A7 in December 2015, which may be ascribed to the direct discharge of wastewater because sites A6 and A7 were near the Yangkou town. The increasing of individual antibiotic in the middle reach (e.g., SMZ) may also be due to the direct discharge of wastewater. Meanwhile, hydrodynamic conditions (e.g., tidal change) in the estuary were complex and may also have an impact on spatial distribution patterns of the total antibiotic concentrations.

Spearman rank correlation analysis showed a negative relationship between TMP and the other six antibiotics, indicating that the pollution discharge or pollution source location of TMP was different from the other six antibiotics. As shown in Fig. 3, an extremely high (> $1000 \mathrm{ng} \mathrm{L}^{-1}$ ) concentration of TMP was detected in the middle and lower reaches of the $\mathrm{XQR}$ estuary in May and September 2015. The results were consistent with previous study (Zhang et al. 2012), which reported that the highest concentration of TMP in the XQR estuary was up to $13,600 \mathrm{ng} \mathrm{L}^{-1}$ in September 2009. The high level of TMP concentration was probably due to the high burden of aquaculture near sites A4-A7. Previous studies have demonstrated that the detection frequency and concentration of TMP in aquaculture zones were high (Graslund and Bengtsson 2001; Zheng et al. 2012). Another possible reason is that pharmaceutical production wastewater enters the middle and/or lower reaches of the XQR estuary via tributary. Although the maximum concentrations of TMP in December 2015 and March 2017 were 2-3 orders of magnitude lower than those in May and September 2015, the relatively high level of TMP still occurred in site A7 or A8 (Fig. 3). However, the location of the maximum concentration of TMP occurred in the XQR estuary was not fixed. For example, the maximum concentration of TMP appeared in site A6 in May 27, 2015, and in site A5 in September 13, 2015, respectively (Fig. 3). The estuarine water is under tidal influence with a change in the water flow direction. The unique hydrodynamic can affect the transport of contaminants, and it may explain some of the variations observed in the spatial distribution of TMP in the XQR estuary. 
Tidal influence on antibiotics in the XQR estuary

\section{Antibiotic concentrations as a function of salinity}

The fate and transport of organic contaminants were more complex in estuarine environments where the hydrodynamic conditions (e.g., water velocity and water direction) and geochemical gradients (e.g., salinity, $\mathrm{pH}$, and turbidity) changed greatly (Du Laing et al. 2009). Generally, organic contaminants in the estuarine environment can react or degrade through physical, chemical, and biological processes (Zhao et al. 2015). If only physical mixing processes occurred, the chemical was conservative in the estuary, and a linear relationship appeared between the concentrations of dissolved chemicals and salinity which usually act as an indicator of the conservative index of mixing. Otherwise, the nonlinearity indicated the non-conservative behavior (Sun et al. 2016). The theoretical dilution line (TDL) was applied to clarify the behavior of antibiotics based on the traditional approaches developed to identify the conservative and non-conservative behaviors in the estuary (Chester 2003). If the concentration point falls onto TDL, it suggests that the dissolved chemical is conservative. If the concentration point is below TDL, it suggests that the dissolved chemical is removed in the estuary through various biogeochemical processes such as adsorption, photolysis, hydrolysis, or biodegradation and transformation. If the concentration point is above TDL, it suggests that the estuary is a source for the dissolved chemical, which is likely caused by additional internal discharge.

The relationships between the seven antibiotic concentrations and salinity are shown in Fig. 4. For TMP, the slope of its TDL was positive, suggesting that its pollution source was located on the lower estuary. On the contrary, the main pollution sources of the other six kinds of antibiotics were located on the upper estuary (i.e., river runoff), as demonstrated by the slopes of their TDL, which were negative. Among the seven
Fig. 4 The relationship between antibiotic concentrations and salinity. The red solid lines represent the theoretic dilution line

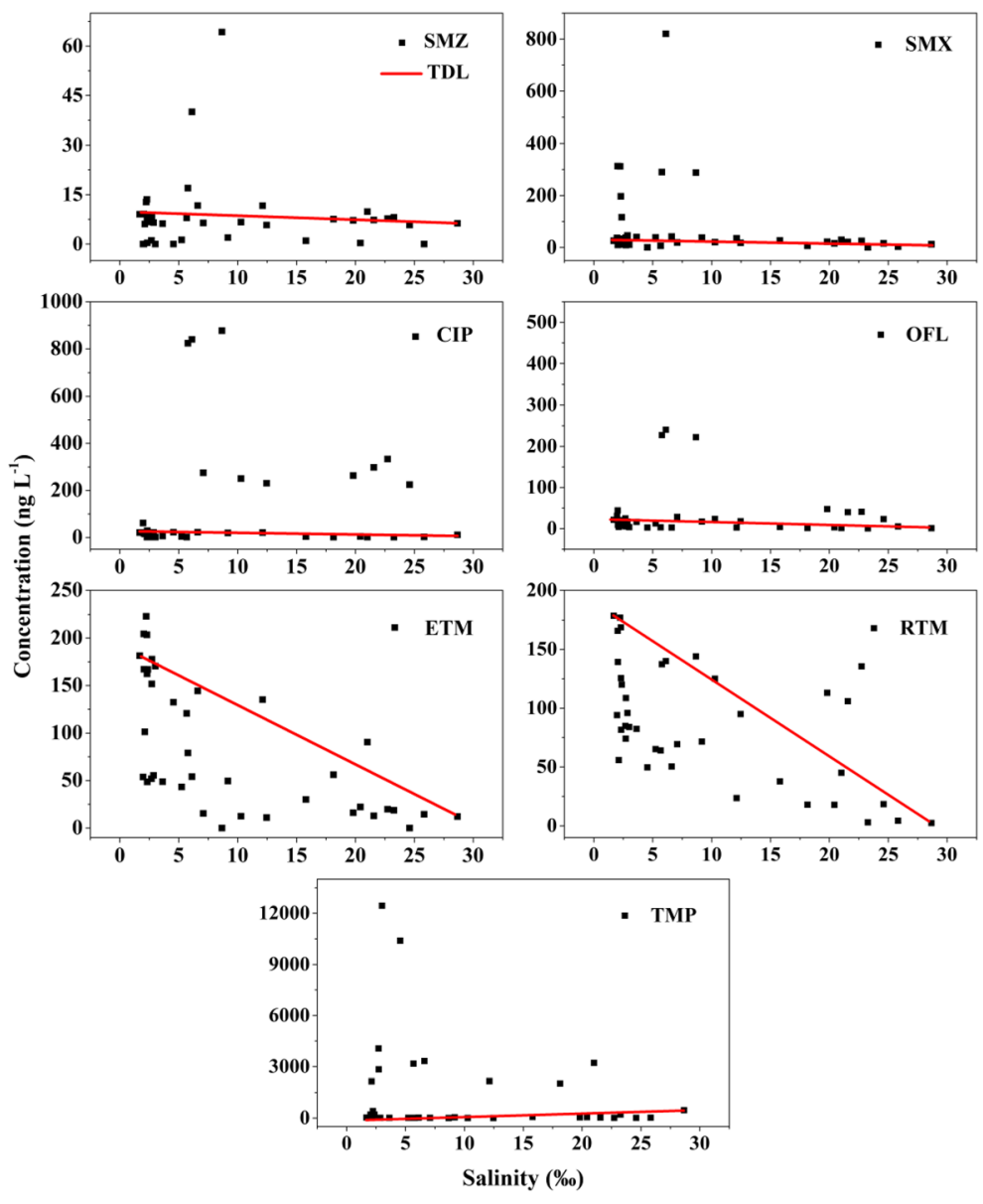


antibiotics, most concentration points (greater than 74\%) of SMZ, SMX, CIP, OFL, and TMP were observed to fall onto either sides of their TDL, indicating that these five antibiotics exhibited conservative behaviors controlled primarily by physical processes. ETM and RTM were found to have most of their concentration points below their TDL, suggesting that their behaviors were non-conservative and these two antibiotics degrade inside estuary. This is reasonable because ETM and RTM are prone to decay in the estuarine environment (Zhao et al. 2015). However, it should be noted that several concentration points are above the TDL of these seven antibiotics, which indicates there are additional internal inputs such as effluent discharge for these antibiotics in the XQR estuary (Chen and Zhou 2014; Zhao et al. 2015). Furthermore, these antibiotics can be adsorbed by suspended particles or sediments (Xu et al. 2009; Li and Zhang 2017; Wang et al. 2017). The release of antibiotics from sediments due to the variations of salinity in the estuarine system may also increase the concentrations of dissolved antibiotics ( $\mathrm{Li}$ and Zhang 2017).

\section{Tidal effects on antibiotic transport in the XQR estuary}

Tides in the estuary can have a great impact on the movement and behaviors of pharmaceuticals (Zhao et al. 2015), especially in the dry season when the river runoff is low. To evaluate the effects of tide on antibiotic transport, we analyzed the continuous variations of antibiotic concentrations at a fixed site (i.e., A6 (Fig. 1)) within $25 \mathrm{~h}$ from noon on May 16, 2017, to noon on May 17, 2017. As shown in Fig. 5, two flood tides and two ebb tides occurred during the sampling period.
Furthermore, the tidal energy of the two high tides was different, as illustrated by the different water levels. The distribution patterns of antibiotic concentrations in the tidal cycle are shown in Fig. 5. From 13:00 to 18:00 on May 16 (i.e., flood tide), the concentration of TMP increased. However, concentrations of the other six kinds of antibiotics decreased sharply, which should be due to pollution discharges. As discussed in the "Antibiotic concentrations as a function of salinity" section, there are internal inputs in the XQR estuary. From 18:00 to 21:00 on May 16 (i.e., ebb tide), the concentrations of TMP and SMZ decreased, and the concentrations of SMX, ETM, and RTM increased, while OFL and CIP decreased first and then increased. From 22:00 on May 16 to 5:00 on May 17 (i.e., flood tide), the concentrations of ETM and RTM decreased, the concentrations of SMZ, SMX, CIP, and OFL fluctuated on a smaller scale, while the concentration of TMP increased obviously. The concentration of TMP decreased again from 6:00 to 12:00 on May 17 (i.e., ebb tide), while the other six kinds of antibiotics increased firstly and then decreased during this period. Overall, the concentrations of the seven antibiotics showed different variations during two tidal cycles.

To further reveal the relationship between antibiotic concentrations and tide, correlation analysis was conducted. As shown in Table S8, there was a significant positive correlation between TMP concentration and the water level $(p<0.01)$, indicating that its concentration increased during the flood tide. However, the concentrations of the other six kinds of antibiotics were negatively correlated with the water level. Among these six antibiotics, the negative correlation between the concentrations of macrolides (i.e., ETM and RTM) and water
Fig. 5 The distribution patterns of antibiotic concentrations in the tidal cycle and water level at site A6. Sampling time is from noon at May 16, 2017, to noon at May 17, 2017

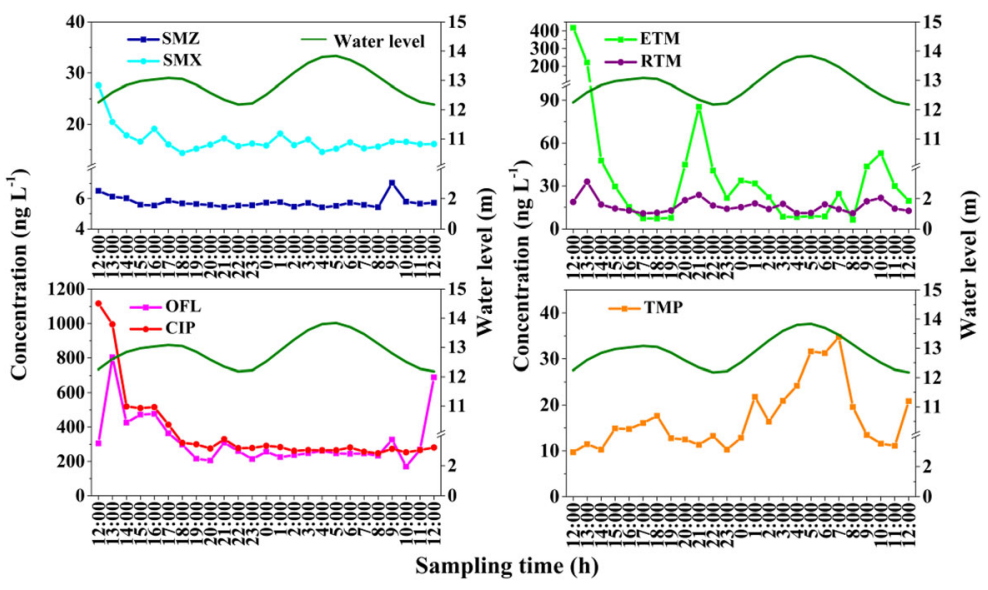


level was significant $(p<0.05)$. This indicated that the concentrations of the six kinds of antibiotics decreased during the flood tide. The different variations of antibiotic concentrations with water level were mainly caused by the location of the pollution sources of different antibiotics. For TMP, its pollution sources were located below the sampling site (i.e., A6). When a flood tide occurred, the polluted water containing high concentration of TMP was pushed to the sampling site, and the TMP concentration at site A6 increased consequently. Meanwhile, when the tidal energy was larger, much more polluted water was pushed to site A6. This is why TMP concentration at the higher tide is higher (Fig. 5). On the contrary, the pollution sources of the other six kinds of antibiotics were located above the site A6. At the low tide, these antibiotics were transported to site A6, resulting in an increase in their concentrations at site A6. At the high tide, the relatively clean seawater pushed these antibiotics back to the upstream of site A6, and therefore, their concentrations at site A6 decreased. In general, tide has impacts on the distribution and transport of antibiotics, especially for TMP, ETM, and $\mathrm{RTM}$, in the XQR estuary. The negative correlation between water level and the concentrations of SMZ, SMX, CIP, and OFL was not significant (Table S8), which may be caused by (1) the relatively large measurement error compared with their concentration range and (2) the impact of other transport mechanisms, e.g., exchange at water-sediment interfaces.

\section{Risk assessment}

In order to elucidate whether these selected antibiotics pose an ecological risk to aquatic organisms in the XQR estuary, the risk of antibiotics to aquatic organisms was assessed based on the RQs, according to the European technical guidance document on risk assessment (EC 2003). The RQs of antibiotics in the XQR estuary were calculated based on the worst-case scenario as a ratio of the maximum measured environmental concentration (MEC) to the predicted no-effect concentration (PNEC). To better evaluate the risk levels, the ratios were classified into three risk levels: RQs $<0.1$, low risk; $0.1 \leq$ $\mathrm{RQs}<1$, medium risk; and $\mathrm{RQs} \geq 1$, high risk (Hernando et al. 2006).

Among the seven antibiotics, only SMZ had low risks to aquatic organisms at four sampling events, as demonstrated by its RQs $<0.1$ (Fig. 6). Although the concentrations of TMP are up to $12,440 \mathrm{ng} \mathrm{L}^{-1}$, it

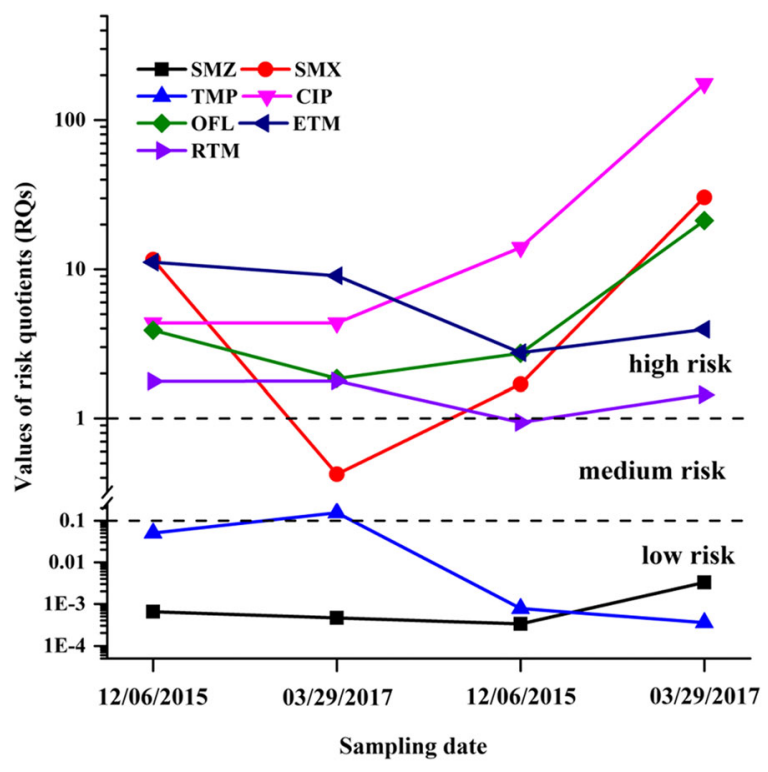

Fig. 6 Values of risk quotients for the seven antibiotics at different sampling date

showed medium risk $(0.1 \leq \mathrm{RQs}<1)$ to the sensitive aquatic species (S. capricornutum) in September 2015 and low risk $(\mathrm{RQs}<0.1)$ in the other three sampling campaigns. By contrast, the other five antibiotics posed relatively higher ecological risks to the relevant aquatic organisms. Especially for CIP, OFL, and ETM, they showed high risks (RQs $\geq 1)$ at all the four sampling events. After entering the wet season (September), the RQs of antibiotics except for TMP decreased in a certain extent. Among them, the risk level of SMX showed a sharp decrease from high to medium risk. Another important phenomenon should be noticed that opening the dam of Wangdao could exacerbate environmental risks of all the seven antibiotics except for TMP. Because the RQ values of the seven antibiotics except for TMP increased after opening the dam, which is in March 2017. Overall, except for SMZ, the other six antibiotics showed medium risk in at least one sampling campaign. Especially in the last sampling, five antibiotics posed high risks for the relevant aquatic organisms. This indicated that the pollution of the targeted antibiotics in the XQR estuary, especially in the upper estuary, was very serious, and regulating their abuse and emission was an urgent task. Nevertheless, it should be noted that the risk assessment above has some limitations, as we could not collect all the toxicity data, and the additional toxicity of the antibiotics on aquatic ecosystem was not considered. 


\section{Conclusions}

In this study, five sampling campaigns were conducted to investigate the occurrence, distribution, transport, and ecological risks of the seven selected antibiotics in the XQR estuary. The results indicated that all the seven antibiotics could be detected in the $\mathrm{XQR}$ estuarine surface water, with a maximum concentration of $12,440 \mathrm{ng} \mathrm{L}^{-1}$. The pollution caused because of the seven antibiotics in the XQR estuary was more serious than that in other estuarine environments reported in literatures. More than five types of antibiotics posed high risks $(\mathrm{RQs} \geq 1)$ for the relevant aquatic organisms in the study area. A relatively high concentration of TMP was detected at the middle or lower reach of the $\mathrm{XQR}$ estuary, while the other antibiotics mainly concentrated in the upper reach, and their concentrations showed a decreasing trend from the XQR estuary to LZB. This implied that most antibiotics in the XQR estuary come from the river runoff, except for TMP. This helps in determining the exact location of the pollution sources of antibiotics in the XQR estuary. Based on the field observation from May 2015 to March 2017, the pollution caused by CIP, OFL, SMX, and RTM became considerably serious, while the concentration of TMP decreased significantly from the wet season to the dry season. Among the seven antibiotics, SMZ, SMX, CIP, OFL, and TMP exhibited conservative behavior being controlled primarily by physical processes, whereas the behaviors of ETM and RTM were non-conservative and exhibited substantial degradation in the estuary. Furthermore, tide has great impacts on the spatial distribution and transport of antibiotics, especially for those with a wide concentration range such as ETM, RTM, and TMP. To provide information for the prevention and control of antibiotic contamination in the XQR estuary, source apportionment of the antibiotics is urgently needed to be conducted in the future.

Funding information This work is supported by the Research Startup Project of Yangzhou University (137011522) and National Nature Science Foundation of China (41271506).

\section{References}

Biel-Maeso, M., Baena-Nogueras, R. M., Corada-Fernandez, C., \& Lara-Martin, P. A. (2018). Occurrence, distribution and environmental risk of pharmaceutically active compounds (PhACs) in coastal and ocean waters from the Gulf of Cadiz (SW Spain). Science of the Total Environment, 612(15), 649-659.
Birch, G. F., Drage, D. S., Thompson, K., Eaglesham, G., \& Mueller, J. F. (2015). Emerging contaminants (pharmaceuticals, personal care products, a food additive and pesticides) in waters of Sydney estuary, Australia. Marine Pollution Bulletin, 97(1-2), 56-66.

Bound, J. P., \& Voulvoulis, N. (2004). Pharmaceuticals in the aquatic environment - a comparison of risk assessment strategies. Chemosphere, 56(11), 1143-1155.

Chapman, P. M., \& Wang, F. Y. (2001). Assessing sediment contamination in estuaries. Environmental Toxicology and Chemistry, 20(1), 3-22.

Chen, K., \& Zhou, J. L. (2014). Occurrence and behavior of antibiotics in water and sediments from the Huangpu River, Shanghai, China. Chemosphere, 95, 604-612.

Cheng, D. L., Ngo, H. H., Guo, W. S., Liu, Y. W., Zhou, J. L., Chang, S. W., Nguyen, D. D., Bui, X. T., \& Zhang, X. B. (2018). Bioprocessing for elimination antibiotics and hormones from swine wastewater. Science of the Total Environment, 621, 1664-1682.

Chester, R., 2003. Marine geochemistry. 2nd edition. Blackwell Science Ltd. (506 pp.).

Cromwell, G. L. (2002). Why and how antibiotics are used in swine production. Animal Biotechnology, 13(1), 7-27.

Du Laing, G., Rinklebe, J., Vandecasteele, B., Meers, E., \& Tack, F. M. G. (2009). Trace metal behaviour in estuarine and riverine floodplain soils and sediments: a review. Science of the Total Environment, 407(13), 3972-3985.

EC, 2003. In: Commission, E. (Ed.), European Commission Technical Guidance Document in Support of Commission Directive 93//67/EEC on Risk Assessment for New Notified Substances and Commission Regulation (EC) No 1488/94 on Risk Assessment for Existing Substance, Part II, pp. 100103.

Graslund, S., \& Bengtsson, B. E. (2001). Chemicals and biological products used in south-east Asian shrimp farming, and their potential impact on the environment - a review. Science of the Total Environment, 280(1-3), 93-131.

Guo, X. Y., Feng, C. H., Zhang, J. H., Tian, C. H., \& Liu, J. L. (2017). Role of dams in the phase transfer of antibiotics in an urban river receiving wastewater treatment plant effluent. Science of the Total Environment, 607-608, 1173-1179.

He, Z., Cheng, X., Kyzas, G. Z., \& Fu, J. (2016). Pharmaceuticals pollution of aquaculture and its management in China. Journal of Moleculor Liquids, 223, 781-789.

Hernando, M. D., Mezcua, M., Fernandez-Alba, A. R., \& Barcelo, D. (2006). Environmental risk assessment of pharmaceutical residues in wastewater effluents, surface waters and sediments. Talanta, 69(2), 334-342.

Hu, S. P., Liu, X. H., \& Peng, Y. (2003). Assessment of antibiotic prescription in hospitalised patients at a Chinese university hospital. Journal of Infection, 46(3), 161-163.

Isidori, M., Lavorgna, M., Nardelli, A., Pascarella, L., \& Parrella, A. (2005). Toxic and genotoxic evaluation of six antibiotics on non-target organisms. Science of the Total Environment, 346, 87-98.

Jiang, L., Hu, X. L., Yin, D. Q., Zhang, H. C., \& Yu, Z. Y. (2011). Occurrence, distribution and seasonal variation of antibiotics in the Huangpu River, Shanghai, China. Chemosphere, 82, 822-828.

Jiang, Y. H., Li, M. X., Guo, C. S., An, D., Xu, J., Zhang, Y., \& Xi, B. D. (2014). Distribution and ecological risk of antibiotics in 
a typical effluent-receiving river (Wangyang River) in north China. Chemosphere, 112, 267-274.

Kim, H. Y., Lee, I. S., \& Oh, J. E. (2017). Human and veterinary pharmaceuticals in the marine environment including fish farms in Korea. Science of the Total Environment, 579(1), 940-949.

Kimosop, S. J., Getenga, Z. M., Orata, F., Okello, V. A., \& Cheruiyot, J. K. (2016). Residue levels and discharge loads of antibiotics in wastewater treatment plants ( WWTPs), hospital lagoons, and rivers within Lake Victoria Basin, Kenya. Environmental Monitoring and Assessment, 188(9), 532.

Li, J., \& Zhang, H. (2017). Factors influencing adsorption and desorption of trimethoprim on marine sediments: mechanisms and kinetics. Environmental Science and Pollution Research, 24(15), 21929-21937.

Li, W. H., Shi, Y. L., Gao, L. H., Liu, J. M., \& Cai, Y. Q. (2012). Occurrence of antibiotics in water, sediments, aquatic plants, and animals from Baiyangdian Lake in North China. Chemosphere, 89, 1307-1315.

Li, J., Zhang, H. B., Chen, Y. S., Luo, Y. M., \& Zhang, H. (2016a). Sources identification of antibiotic pollution combining land use information and multivariate statistics. Environmental Monitoring and Assessment, 188(7), 430.

Li, J., Zhang, R. J., Wang, R. M., Zhang, H., Jiang, D. J., Zou, T., Tang, J. H., \& Lu, J. (2016b). Distribution characteristics and ecological risk assessment of antibiotic pollution in Xiaoqing River watershed. Journal of Agro-Environment Science, 35(7), 1384-1391.

Lindholm-Lehto, P. C., Ahkola, H. S. J., Knuutinen, J. S., \& Herve, S. H. (2016). Widespread occurrence and seasonal variation of pharmaceuticals in surface waters and municipal wastewater treatment plants in central Finland. Environmental Science and Pollution Research, 23(8), 7985-7997.

Liu, S., Zhao, H., Lehmler, H. J., Cai, X., \& Chen, J. (2017). Antibiotic pollution in marine food webs in Laizhou Bay, North China: trophodynamics and human exposure implication. Environmental Science \& Technology, 51(4), 23922400.

Loos, R., Carvalho, R., Antonio, D. C., Cornero, S., Locoro, G., Tavazzi, S., Paracchini, B., Ghiani, M., Lettieri, T., Blaha, L., Jarosova, B., Voorspoels, S., Servaes, K., Haglund, P., Fick, J., Lindberg, R. H., Schwesig, D., \& Gawlik, B. M. (2013). EU-wide monitoring survey on emerging polar organic contaminants in wastewater treatment plant effluents. Water Research, 47(17), 6475-6487.

Lunestad, B. T., Samuelsen, O. B., Fjelde, S., \& Ervik, A. (1995). Photostability of 8 antibacterial agents in seawater. Aquaculture, 134(3-4), 217-225.

Luo, Y., Xu, L., Rysz, M., Wang, Y. Q., Zhang, H., \& Alvarez, P. J. J. (2011). Occurrence and transport of tetracycline, sulfonamide, quinolone, and macrolide antibiotics in the Haihe River Basin, China. Environmental Science \& Technology, 45(5), 1827-1833.

Luo, X., Zhang, S., Yang, J., Pan, J., Tian, L., \& Zhang, L. (2013). Macrobenthic community in the Xiaoqing River Estuary in Laizhou Bay, China. Journal of Ocean University of China, 12(3), 366-372.

Mcardell, C. S., Molnar, E., Suter, M. J., \& Giger, W. (2003). Occurrence and fate of macrolide antibiotics in wastewater treatment plants and in the Glatt Valley Watershed, Switzerland. Environmental Science \& Technology, 37(24), 5479-5486.

Ngigi, A. N., Magu, M. M., \& Muendo, B. M. (2020). Occurrence of antibiotics residues in hospital wastewater, wastewater treatment plant, and in surface water in Nairobi County, Kenya. Environmental Monitoring and Assessment, 192(1), 18.

Shen, J., Luo, X., Zheng, H., Yang, J., Lin, S., Zhang, J., \& Zhang, S. (2017). Pollution and ecological risk characteristics of heavy metals in surface sediments in Xiaoqing River Estuary and adjacent sea areas. Environmental Chemistry, 36(7), 1516-1524.

Shi, Y. L., Vestergren, R., Xu, L., Song, X. W., Niu, X. M., Zhang, C. H., \& Cai, Y. Q. (2015). Characterizing direct emissions of perfluoroalkyl substances from ongoing fluoropolymer production sources: a spatial trend study of Xiaoqing River, China. Environmental Pollution, 206, 104-112.

Simpson, J. H., Vennell, R., \& Souza, A. J. (2001). The salt fluxes in a tidally-energetic estuary. Estuarine Coastal and Shelf Science, 52(1), 131-142.

Spielmeyer, A., Hoeper, H., \& Hamscher, G. (2017). Long-term monitoring of sulfonamide leaching from manure amended soil into groundwater. Chemosphere, 177, 232-238.

Sun, Q., Li, Y., Li, M., Ashfaq, M., Lv, M., Wang, H., Hu, A., \& Yu, C. P. (2016). PPCPs in Jiulong River estuary (China): spatiotemporal distributions, fate, and their use as chemical markers of wastewater. Chemosphere, 150, 596-604.

Tamtam, F., Mercier, F., Le Bot, B., Eurin, J., Dinh, Q. T., Clement, M., \& Chevreuil, M. (2008). Occurrence and fate of antibiotics in the Seine River in various hydrological conditions. Science of the Total Environment, 393(1), 84-95.

Vieno, N. M., Tuhkanen, T., \& Kronberg, L. (2005). Seasonal variation in the occurrence of pharmaceuticals in effluents from a sewage treatment plant and in the recipient water. Environmental Science \& Technology, 39, 8220-8226.

Vo, T. D. H., Bui, X. T., Cao, N. D. T., Luu, V. P., Nguyen, T. T., Dang, B. T., Thai, M. Q., Nguyen, D. D., Nguyen, T. S., Dinh, Q. T., \& Dao, T. S. (2016). Investigation of antibiotics in health care wastewater in Ho Chi Minh City, Vietnam. Environmental Monitoring and Assessment, 188(12), 686.

Wang, P., Zhang, D., Zhang, H., Li, H., Ghosh, S., \& Pan, B. (2017). Impact of concentration and species of sulfamethoxazole and ofloxacin on their adsorption kinetics on sediments. Chemosphere, 175, 123-129.

Wolf, A., Bergmann, A., Wilken, R. D., Gao, X., Bi, Y., Chen, H., \& Schueth, C. (2013). Occurrence and distribution of organic trace substances in waters from the Three Gorges Reservoir, China. Environmental Science and Pollution Research, 20(10), 7124-7139.

Xu, W. H., Zhang, G., Wai, O. W. H., Zou, S. C., \& Li, X. D. (2009). Transport and adsorption of antibiotics by marine sediments in a dynamic environment. Journal of Soils and Sediments, 9(4), 364-373.

Yan, C. X., Yang, Y., Zhou, J. L., Liu, M., Nie, M. H., Shi, H., \& Gu, L. J. (2013). Antibiotics in the surface water of the Yangtze Estuary: occurrence, distribution and risk assessment. Environmental Pollution, 175, 22-29.

Yang, Y., Liu, W., Xu, C., Wei, B., \& Wang, J. (2017). Antibiotic resistance genes in lakes from middle and lower reaches of 
the Yangtze River, China: effect of land use and sediment characteristics. Chemosphere, 178, 19-25.

Zhang, R. J., Zhang, G., Zheng, Q., Tang, J. H., Chen, Y. J., Xu, W. H., Zou, Y. D., \& Chen, X. X. (2012). Occurrence and risks of antibiotics in the Laizhou Bay, China: impacts of river discharge. Ecotoxicology and Environmental Safety, 80, 208-215.

Zhang, Q. Q., Ying, G. G., Pan, C. G., Liu, Y. S., \& Zhao, J. L. (2015). Comprehensive evaluation of antibiotics emission and fate in the river basins of China: source analysis, multimedia modeling, and linkage to bacterial resistance. Environmental Science \& Technology, 49(11), 6772-6782.

Zhang, R. J., Zhang, R. L., Li, J., Cheng, Z. N., Luo, C. L., Wang, Y. H., Yu, K. F., \& Zhang, G. (2017). Occurrence and distribution of antibiotics in multiple environmental media of the East River (Dongjiang) catchment, South China. Environmental Science and Pollution Research, 24(10), 9690-9701.

Zhang, R. L., Pei, J. Y., Zhang, R. J., Wang, S. P., Zeng, W. B., Huang, D. L., Wang, Y., Zhang, Y. Y., Wang, Y. H., \& Yu, K. F. (2018a). Occurrence and distribution of antibiotics in mariculture farms, estuaries and the coast of the Beibu Gulf, China: bioconcentration and diet safety of seafood. Ecotoxicology and Environmental Safety, 154, 27-35.

Zhang, R. J., Zhang, R. L., Yu, K. F., Wang, Y. H., Huang, X., Pei, J., Wei, C., Pan, Z. L., Qin, Z., \& Zhang, G. (2018b). Occurrence, sources and transport of antibiotics in the surface water of coral reef regions in the South China Sea: potential risk to coral growth. Environmental Pollution, 232, 450-457.

Zhao, H., Zhou, J. L., \& Zhang, J. (2015). Tidal impact on the dynamic behavior of dissolved pharmaceuticals in the
Yangtze Estuary, China. Science of the Total Environment, 536, 946-954.

Zhao, Z. L., Wang, J., Han, Y., Chen, J. W., Liu, G. F., Lu, H., Yan, B., \& Chen, S. S. (2017). Nutrients, heavy metals and microbial communities co-driven distribution of antibiotic resistance genes in adjacent environment of mariculture. Environmental Pollution, 220, 909-918.

Zheng, Q., Zhang, R. J., Wang, Y. H., Pan, X. H., Tang, J. H., \& Zhang, G. (2012). Occurrence and distribution of antibiotics in the Beibu Gulf, China: impacts of river discharge and aquaculture activities. Marine Environmental Research, 78, 26-33.

Zheng, J., Gao, R. X., Wei, Y. Y., Chen, T., Fan, J. Q., Zhou, Z. C., Makimilua, T. B., Jiao, Y. N., \& Chen, H. (2017). Highthroughput profiling and analysis of antibiotic resistance genes in East Tiaoxi River, China. Environmental Pollution, 230, 648-654.

Zou, S. C., Xu, W. H., Zhang, R. J., Tang, J. H., Chen, Y. J., \& Zhang, G. (2011). Occurrence and distribution of antibiotics in coastal water of the Bohai Bay, China: impacts of river discharge and aquaculture activities. Environmental Pollution, 159, 2913-2920.

Zou, T., Zhang, H., Meng, Q. J., \& Li, J. (2016). Seasonal hydrodynamics and salt exchange of a shallow estuary in northern China. Journal of Coastal Research, 74, 95-103.

Publisher's note Springer Nature remains neutral with regard to jurisdictional claims in published maps and institutional affiliations. 